\title{
EVALUATION OF THE ANTIDEPRESSANT EFFECTS OF ALCOHOLIC EXTRACTS OF PILEA MICROPHYLLA IN MICE
}

\author{
DARAH IBRAHIM ${ }^{1}$, AMIR MODARRESI CHAHARDEHI ${ }^{2 *}$, \\ FARID ABOLHASSANI ${ }^{3} \&$ SHAIDA FARIZA SULAIMAN ${ }^{4}$
}

\begin{abstract}
To date, the search for novel pharmacotherapy from medicinal plants for psychiatric illnesses has significantly progressed. This study investigated the effect of selected crude extracts from Pilea microphylla in the mouse forced test (FST) and in the tail suspension test (TST), two models predictive of antidepressant activity. Selected crude extracts from Pilea microphylla produced an antidepressant-like effect, since the acute treatment of mice with extracts by intraperitoneal (i.p.) route significantly reduced the immobility time in the FST (50 and 100 $\mathrm{mg} / \mathrm{kg}$ ) and TST (50 and $100 \mathrm{mg} / \mathrm{kg}$ ), as compared to positive controls (haloperidol and fluoxetine) at 1 and $10 \mathrm{mg} / \mathrm{kg}$, respectively. The antidepressant-like effect of extracts was found to be significant at high doses, followed by an increase in the immobility time at dose of $100 \mathrm{mg} / \mathrm{kg}$. A significant decreased of immobility was also found on the third day at the concentration of 100 $\mathrm{mg} / \mathrm{kg}$ of chloroform extract of Pilea microphylla from extraction method II (CEPM II) and ethyl acetate extract of Pilea microphylla from extraction method II (EAEPM II); (except methanol extract of Pilea microphylla from extraction method I (MEPM I) at $100 \mathrm{mg} / \mathrm{kg}$ ) with respect to the first day. Ethyl acetate and chloroform extract from extraction method II when administered at an acute dose of $100 \mathrm{mg} / \mathrm{kg}$ of body weight $(\mathrm{P}<0.05)$ reduced the immobility time. Among all the three selected extracts with two doses administered there were differences compared to the control, EAEPM II led to reduction of immobility time, in the FST method by $38.50 \%$ for 50 $\mathrm{mg} / \mathrm{kg}$ to as much as $75.97 \%$ for $100 \mathrm{mg} / \mathrm{kg}$. Similar results of increased antidepressant effect, that was, of immobility time depending on the concentration administered, were obtained with the TST method. These results suggested the anti-depression activity of the plant extract. Therefore, P. microphylla may be served as a potential resource for natural psychotherapeutic agent against depression. However, further studies are still required.
\end{abstract}

Keywords: Pilea microphylla; antidepressant activity; forced swimming test (FST); tail suspension test (TST)

1:2 Industrial Biotechnology Research Laboratory, School of Biological sciences, Universiti Sains Malaysia, 11800 Minden, Penang, Malaysia

${ }^{3}$ Department of Anatomy, Medical School, Tehran University of Medical Sciences, Iran

4* 5 Phytochemistry Laboratory, School of Biological Sciences, Universiti Sains Malaysia, 11800

Minden, Penang, Malaysia

* Corresponding author: amirmch@yahoo.com 


\subsection{INTRODUCTION}

Depression is a common, debilitating, life-threatening illness with a significant incidence in the population. Numerous antidepressant compounds are now available, presumably acting via different mechanisms including serotonergic, noradrenergic and/or dopaminergic systems. Heterogeneity of the clinical responses to antidepressant and mood-stabilizing drugs and susceptibility to adverse effects are the major clinical problems [1]. Some other chemical medicine has side effects such as fluoxetine causes urinary retention and extrapyramidal symptoms during treatment [2].

Interactions between monoamine neurotransmitters system including 5hydroxytryptamine (5-HT), noradrenaline (NA) and dopamine (DA) in the brain along with their specific reuptake and receptor protein has gain so much interest in the spectrum of antidepressant studies [3].

The hypothalamic-pituitary-adrenal (HPA) axis in the neuroendocrine system is one of the complicated neurobiological mechanisms which play important roles as similar as monoamine neurotransmitters system in the new antidepressant development. Dysfunction or hyperactivity of HPA axis system provides significant indicator of depression together in the response to stressors reflected by overproduction of glucocorticoid hormones mainly corticosterone in rodent and cortisol in human [4]. Hence, the normalization of the HPA axis system as another prime mechanism of antidepressant actions appears to be one of the special interest [5].

Herbal therapy is another effective alternative to treat depression. The search for novel therapeutic natural plants that mitigate depressive illness has been extensively explored over the past decade [6,7]. There are also a large number of herbal medicines whose therapeutic potential have been assessed in a variety of animal models [6]. Often, the models are based on the application of stress to the animal, as in the forced swim test (FST) [8] or the tail suspension test (TST) [9]. FST and TST are widely used for screening potential antidepressants. Antidepressants reduce the immobility time in both FST and TST. The immobility behavior displayed in rodents when subjected to an unavoidable and inescapable stress has been hypothesized to reflect behavioral despair which in turn may reflect depressive disorders in humans. There is, indeed, a significant correlation between clinical potency and effectiveness of antidepressants in both models [10]. 
Our previous study showed that this plant had antioxidant, antimicrobial activity $[11,12]$ and also anticancer activity (data not published). According to our previous data, we selected some best crude extract namely, methanol extract from extraction method I, chloroform and ethyl acetate extracts from extraction method II for antidepressant activity on mice. This study sought to investigate the effect of selected crude extracts from two methods of extractions of this plant in FST and TST, predictive models of antidepressant activity. Also to explore if the immobility (it might be supposedly learned) can be forgotten as a consequence of the passage of time by means of increasing the time intervals (more than one time experiment). In this study, a variation of the original Porsolt test was used [8], which means placing the mice in the water tank in two occasions separated by different days and recording swimming activity automatically.

\subsection{MATERIALS AND METHODS}

\subsection{Plant Material and Extraction}

The whole parts of Pilea microphylla were collected in Penang island from USM main campus (Universiti Sains Malaysia) in March 2008. Voucher specimens have been deposited at the Herbarium of the School of Biological Sciences, Universiti Sains Malaysia in April 2008. The plant materials were washed, dried and ground to small pieces. The first method (Method I) of extraction included the using of four solvents by following non-polar to polar solvents (by using Soxhlet apparatus). In this method, dried powdered plant was extracted. The solvents used were hexane, chloroform, ethyl acetate and methanol. The second method (Method II) included 5 solvents system (by using partition technique). For Method II, the dried materials were extracted by using soxhlet extractor with methanol as a solvent for 72 hours at room temperature $\left(30^{\circ} \mathrm{C}\right)$. The methanolic extracts were further partitioned by adding distilled water in a separating funnel and then followed using chloroform, diethyl ether, ethyl acetate and butanol as described by Mellidis and Papageorgiou [13], with a slight modification. The dried extracts were then weighed using microbalance and were kept at $4 \mathrm{C}$. Abbreviations for crude extract used in this paper namely:

HEPM I (hexane extract of method I), CEPM I (chloroform extract of method I), EAEPM I (ethyl acetate extract of method I), MEPM I (methanol extract of 
method I), ME II (methanol extract of method II), CE II (chloroform extract of method II), DEE II (diethyl ether extract of method II), EAE II (ethyl acetate extract of method II) and BE II (butanol extract of method II).

According to our previous studies in our lab, antioxidant, antimicrobial, toxicity and anticancer activities, three of these crude extracts (MEPM I, CEPM II and EAEPM II) were selected for antidepressant activity.

\subsection{Animals}

Mice of either sex purchased from animal house, School of Medical Sciences, University of Tehran (Iran), and weighing 25-35 g were used. Animals were placed at Animal house, Department of Anatomy, School of Medical Sciences, University of Tehran, housed 6 per cage under a normal 12 h/12 h light/dark schedule with the lights on at 07:00 a.m. and had free access to water and food pellets. They were allowed at least 1 week to adapt to the laboratory prior to the administration. All efforts were made to minimize animal suffering and to reduce the number of animal used. All the drugs were administered intraperitoneally (i.p.) 30 min prior to FST.

\subsection{Forced Swimming Test (FST)}

Mice of either sex were individually forced to swim in an open cylindrical container (diameter $10 \mathrm{~cm}$, height $25 \mathrm{~cm}$ ), containing $19 \mathrm{~cm}$ of water at $25 \pm 1 \mathrm{C}$. The immobility time, defined as the absence of escape-oriented behaviors, such as swimming, was scored during $6 \mathrm{~min}$ with the help of stop-watch [14-16]. All the mice of either sex were divided in five different groups. The first group assigned as control receiving only vehicle $(\mathrm{NaCl} 5 \mathrm{ml} / \mathrm{kg})$. The other groups received acute dose of extracts $(50,100 \mathrm{mg} / \mathrm{kg})$. The positive group received standard drugs such as Haloperidol (diluted with sterile water) and Fluoxetine (diluted with normal saline) (1 and $10 \mathrm{mg} / \mathrm{kg}$, respectively). The total duration of immobility was recorded during the last $6 \mathrm{~min}$ of the 10-min period. Each mouse was judged to be immobile when it ceased struggling and remained floating motionless in the water, making only those movements necessary to keep its head above water. A decrease in the duration of immobility is indicative of an antidepressant like effect. This 
experiment was injected for two days later with same the doses of selected crude extracts of P. microphylla (Third day) with a bit modification [17].

\subsection{Tail Suspension Test (TST)}

The total duration of immobility induced by tail suspension test was measured according to the method described by Steru et al. [9]. Mice both acoustically and visually isolated were suspended $70 \mathrm{~cm}$ above the floor by adhesive tape placed approximately $1 \mathrm{~cm}$ from the tip of the tail. The total immobility period was scored manually during 6 minutes test session with the help of stopwatch. Immobility was defined as the absence of any limb or body movements, except for those caused by respiration or when they hung passively and completely motionless. The parameter obtained was the number of seconds spent immobile. Parameter used was the number of seconds spent immobile.

\subsection{Statistical Analysis}

Data were expressed as the mean \pm standard deviation of mean (S.D.). Comparisons between experimental and control groups were performed by oneway analysis of variance (ANOVA) followed by Tukey`s HSD test when appropriate. $\mathrm{P}<0.05$ was considered significant.

\subsection{RESULTS AND DISCUSSIONS}

The results presented here showed that the selected crude extract of $P$. microphylla given systemically (i.p. route), are effective in producing significant antidepressant-like effects, when assessed in the FST and in the TST. The antidepressant-like effects of these extracts in the FST and TST were comparable to haloperidol and fluoxetine as positive controls.

Behavioral studies have been shown to play an important part in the evaluation and development of antidepressant drugs [4]. Forced swimming test (FST) and tail suspension test (TST) are among behavioral models that widely and routinely used for screening new antidepressant compound [18]. In this study, statistically 
significant results were obtained in FST with treatment of mostly selected crude extracts of Pilea microphylla. Positive control antidepressant drugs haloperidol and fluoxetine produced significant result on immobility time $(21.66 \%$ and $56.49 \%$ reduction respectively in FST test). Otherwise, according to study by Karolewicz and Paul [19], we also housed mice in groups (six per cage) displayed high levels of immobility in both the FST and TST methods.

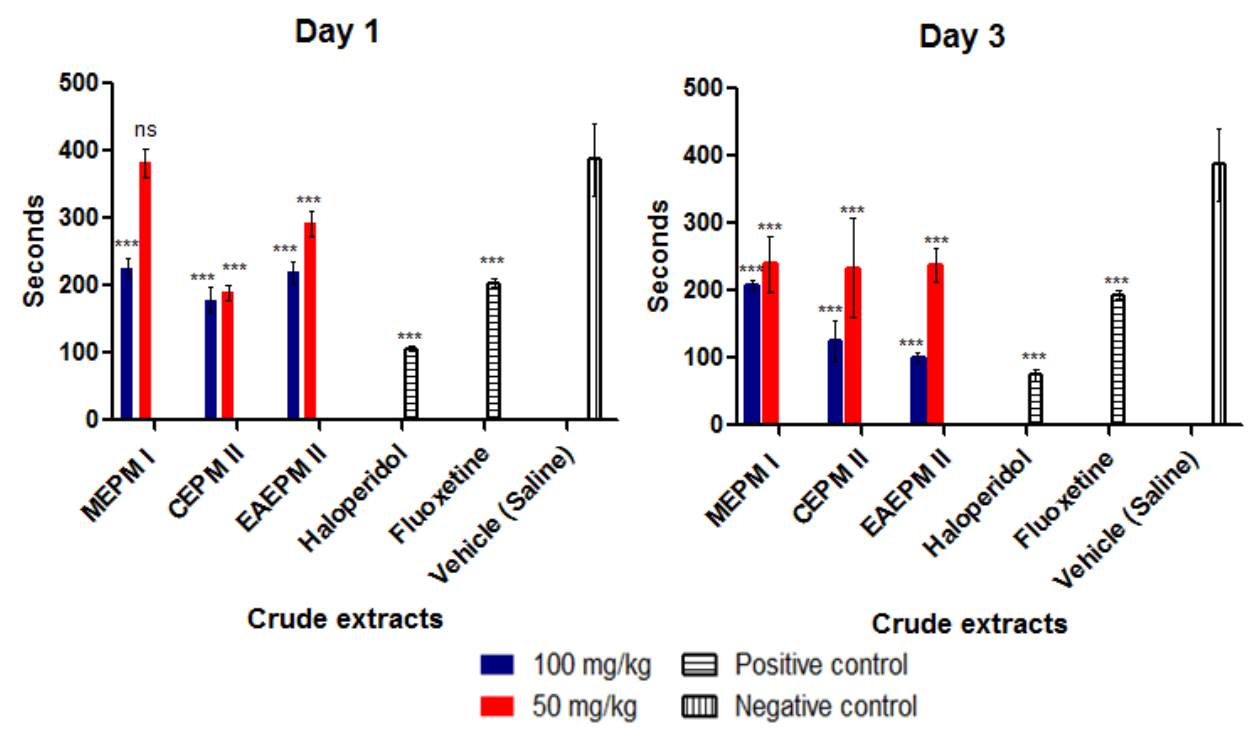

Figure 1 Antidepressant activity of crude extracts of Pilea microphylla from Forced swimming test (FST). Values were mean \pm SD $\left(n=3\right.$ mice). ${ }^{\prime \prime}$ Data are significantly different with negative control $(\mathrm{P}<0.05)$, ns $=$ Data are not different significant

The results obtained after a single administration of extracts suspension showed that the immobility time of animals decreased dose-dependently, namely, the animals were more active in both employed models, which means that the antidepressant effect were stronger. For all two doses administered there were differences compared to our controls, that are, led to reduction of immobility time, in the FST method for MEPM I at 50 and $100 \mathrm{mg} / \mathrm{kg}$ were $38.17 \%$ and $46.18 \%$, for CEPM II at 50 and $100 \mathrm{mg} / \mathrm{kg}$ were $39.62 \%$ and $67.70 \%$, and for EAEPM II at 50 and $100 \mathrm{mg} / \mathrm{kg}$ were $38.50 \%$ and $75.97 \%$ (Figure 1). Also the reduction time in the TST method for MEPM I at 50 and $100 \mathrm{mg} / \mathrm{kg}$ showed 
$42.39 \%$ and $55.16 \%$, for CEPM II at 50 and $100 \mathrm{mg} / \mathrm{kg}$ were $-8 \%$ (or no effect) and $23.41 \%$, and for EAEPM II at 50 and $100 \mathrm{mg} / \mathrm{kg}$ were $22.39 \%$ and $77.61 \%$.

Mice pretreated with varying doses of MEPM I, CEPM II and EAEPM II to forced swim showed no statistically reliable alterations between the first of day of forced swim exposure and third day except MEPM I at $50 \mathrm{mg} / \mathrm{kg}$ on the first day (Figure 1, panel left).

The highest dose of selected crude extracts of Pilea microphylla such as CEPM II, EAEPM II showed comparable effect as fluoxetine and haloperidol in terms of its antidepressant actions revealing these crude extracts might share some pharmacological mechanisms with established antidepressant drugs in this investigation.

However, statistical significant reduction in mean immobility time during TST was observed with doses of $100 \mathrm{mg} / \mathrm{kg}$ for EAEPM II (Figure 2).

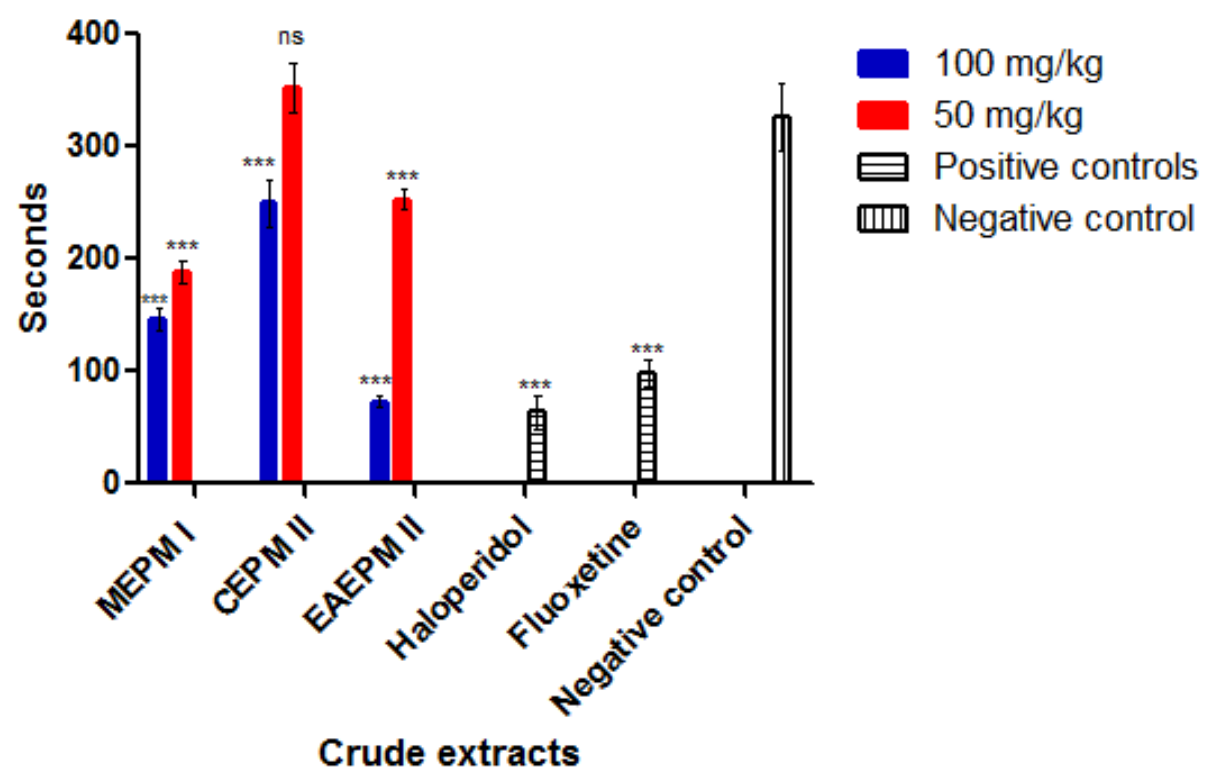

Figure 2 Tail suspension test from some selected crude extracts of Pilea microphylla. Values were mean $\pm \mathrm{SD}$ ( $\mathrm{n}=3$ mice). '” Data are significantly different with negative control (P $<0.05)$, ns $=$ Data are not different significant

For further process, we selected CEPM II extract for fractionation (according to its high extraction yield (\%), antimicrobial, anticancer activities). EAEPM II also 
showed best results, due to achieve low yield of extraction, we did not follow further with this extract.

According to polarity of CEPM II crude extract to obtain terpenoids and flavonols as the major constituent was found. As shown in Figure 3, in TLC chromatogram of CEPM II to get fractionation using EtOAc: n-Hexane (4:16) under UV light, flavonols at $98.68 \mathrm{R}_{\mathrm{r}}$ value was appeared at end of TLC in bright yellow spots in UV light and without colour in visible light. Some flavonoids like biflavones were appeared in high $\boldsymbol{R}_{\mathrm{f}}$ value. Flavones are widespread in leaves [20]. Most bands in UV light were appeared in red and dark red colour. According to Harborne [20], they are phenolic compounds and dark red or bright orange in UV light one of the colour properties of flavonoid in ultraviolet light, although in visible colour these spot in bright yellow.

Majority of bands under visible light represented in green colour except the last band (eighth band) appeared in yellow colour. This band could be in flavonols group. Flavonols are appeared in bright yellow and bright yellow fluorescent in high $R_{\mathrm{r}}$ value. Although some highly methylated flavonols behave similarly [20].

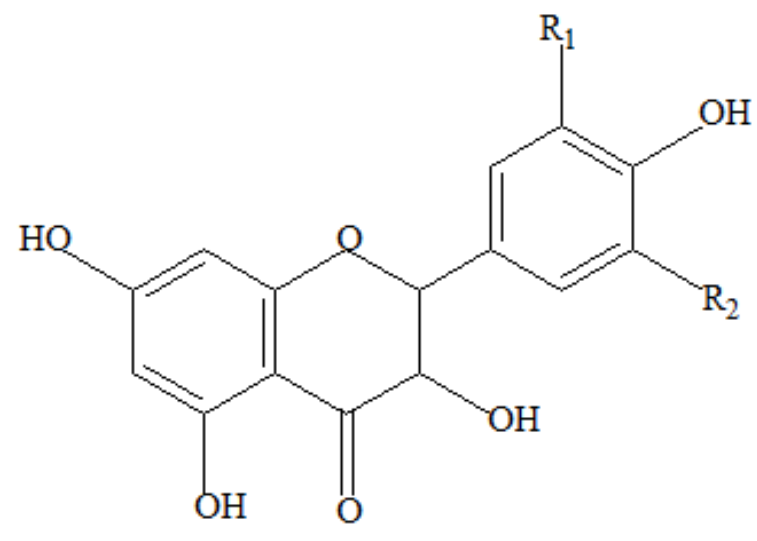

Figure 3 Some constituents derived from Urticaceae family involved in antidepressant-like effect. $\left(\mathrm{R}_{1}=\mathrm{H} ; \mathrm{R}_{2}=\mathrm{H}\right.$ : Kaempferol, $\mathrm{R}_{1}=\mathrm{OH} ; \mathrm{R}_{2}=\mathrm{H}$ : Quercetin, $\mathrm{R}_{1}=\mathrm{OH} ; \mathrm{R}_{2}=\mathrm{OH}$ : Myricetin and $\mathrm{R}_{1}=\mathrm{OCH}_{3} ; \mathrm{R}_{2}=\mathrm{H}$ : Isorhamnetin) 
In general, several classes of compounds have been found in members of this family. These are the proanthocyanidins and flavonols. When the proanthocyanidins are present, cyanidin is the compound identified. When the flavonols are present, quercetin is commonly found. Sometimes, both kaempferol and quercetin can be found. Ellagic acid has been reported to be absent in eight species and five genera. In addition, arbutin was absent and aluminium accumulation was not found [21]. Recently, several studies have suggested the antidepressant effect of quercetin glycosides such as hyperoside, isoquercitin and rutin using the positive results of FST. Although, the plant contains kaempferol and rutin. These flavonoid glycosides seem to appear as conjugated forms in the blood stream as with quercetin glycosides. Transportation of these metabolites into the brain tissues via the blood brain barrier and their effect on the CNS system has been recently argued [22]. In study by Paulke et al. [23] reported that quercetin metabolites were previously found in the brain tissues of rodents after oral administration. It seems one of the antidepressant mechanisms of $P$. microphylla is thought to involve flavonoid glycosides, that reach the brain tissues through to involve flavonoid glycosides, which reach the brain tissues through the metabolizing process, protecting brain function from CNS disturbance, and consequently, exerting an antidepressant effect.

The FST and TST are well established screening paradigms for antidepressant. Although, the TST, a derivative of the FST, shares with the FST the ability to induce a state of immobility in animals, which is claimed to reproduce a condition akin to human depression [24,8-9]. However, the immobility displayed by rodents when subjected to unavoidable stress such as forced swimming is thought to reflect a state of despair or lowered mood, which are thought to reflect depressive disorders in humans. In addition, the immobility time has been shown to be reduced by treatment with antidepressant drugs. Moreover, a significant correlation was found between the clinical efficacy of antidepressant drugs and their potency in both models [8,22].

Although the mean value of immobility time for the control group of animals was higher in the forced swimming test than in the tail suspension test, this difference was not significant. However, when comparing the changes of immobility time in extract-treated animals, expressed as the percent of control values, between two tests, the reduction of immobility time was more marked in the FST method. This means that FST is more sensitive and better reflects the state of depression [25]. 
Forced swimming in FST induced alterations in the HPA axis, thereby increase the cortisol level of mice [26]. Porsolt et al. proposed this behavioral model for the screening of new antidepressant compounds, concluded that the immobility time observed in the test reflected a state of lowered mood or hopelessness in animals, thus, this animal model is the most widely used tool for preclinical screening of putative antidepressant agents $[27,18]$.

The involvement of 5 - $\mathrm{HT}_{3}$ receptors in the pathophysiology of depression is less reported in the literature, but some reports have indicated that different classes of antidepressants act as functional antagonists at the 5-HTs receptors, indicating that the suppression of $5-\mathrm{HT}_{3}$ receptor activity may contribute to the action of antidepressants [28].

Fluoxetine is a selective serotonin reuptake inhibitor (SSRI) [29], while the mechanism of action of haloperidol has not been entirely elucidated, but has been attributed to the inhibition of the transport mechanism of cerebral monoamines, particularly by blocking the impulse transmission in dopaminergic neurons [30]. However, Fluoxetine (Prozac) in pharmacologic treatments for depression in cancer patients at doses 10-20 $\mathrm{mg}$ as starting dose and 20-60 $\mathrm{mg}$ as therapeutic range has common side-effect such as varying degrees of gastrointestinal [31].

Rocha et al. [32] reported that Cecropia glazioui from Urticaceae family as same as P. microphylla, had antidepressant-like effect. The effect was enhanced after purification of the active extract. Catechins, procyanidins and flavonoids were the main constituents of the purified active fraction. So far, in vitro studies showed that catechin $(4 \alpha \rightarrow 8)$ ent-catechin (Procyanidiin B3 isomer), catechin and epicatechin $(4 \alpha \rightarrow 8)$ epicatechin (Procyanidin B2) inhibited 5-HT, NA and DA uptakes in brain synaptosomes but the flavonoids isoorientin and isovitexin did not. Rocha et al. found that chatechin and procyanidins are the major active substances in vitro and may contribute to the antidepressant-like effect produced by $C$. glazioui.

However, some consideration should be taking into account that FST and TST is not the only model of depression by which the results obtained using this model should be considered and interpreted with caution due to some differences among experimental animals and clinical studies in human [5]. It has been reported that the TST is less stressful than FST and has greater pharmacological sensitivity. Remarkably, TST detects the anti-immobility effects of a wide array of antidepressants. Thus, the activity of $P$. microphylla could involve one of the mechanisms of the antidepressant. 


\subsection{CONCLUSION}

In conclusion, the present study indicates that Pilea microphylla produces a specific antidepressant-like effect in animal models predictive of antidepressant properties, forced swimming test and tail suspension test. Moreover, the effect of the acute or repeated administration of this extract was similar to the action produced by the classical antidepressant fluoxetine and haloperidol.

\section{AGKNOWLEDGEMENT}

This material is based upon work supported by a grant from Universiti Sains Malaysia (USM). We also thank USM because this research was partially supported by the provided fellowship. Also we thank Dr. Kashani, the head of Embryology lab in School of Medical Sciences in University of Tehran and Ms. Soudabeh Ghasemi for her support.

\section{REFERENCES}

[1] B. Lerer, and F. Macciardi. 2002. Pharmacogenetics of Antidepressant and Moodstabilizing Drugs: A Review of Candidate-Gene Studies and Future Research Directions. International Journal of Neuropsychopharmacology. 5: 255-275.

[2] G. Bondolfi, C. B. Eap, G. Bertschy, D. Zullino, A. Vermeulen, and P. Baumann. 2002. The Effect of Fluoxetine on the Pharmacokinetics and Safety of Riseperidone in Psychiatric Patients. Pharmacopsychiatry. 35: 50- 56.

[3] L. T. Yi, J. M. Li, Y. C. Li, Y. Pan, Qun. Xu, and L. D. Kong. 2008. Antidepressant-like BehavIoral and Neurochemical Effects of the Citrus-Associated Chemical Apigenin. Life Sci. 82: 741751.

[4] Q. Xu, L. T. Yi, Y. Pan, X. Wang, Y. C. Li, J. M. Li, C. P. Wang, and L. D. Kong. 2008. Antidepressant-like Effects of mixture of Honokio Land Magnolol from the Barks of Magnolia Officinalis In Stressedrodents. Prog. Neuropsychopharmacol. Biol. Psychiatry. 32: 715 -725.

[5] N. Farah Idayu, M. Taufik Hidayat, M. A. M. Moklas, F. Sharida, A. R. Nurul Raudzah, A. R. Shamima, and E. Apryani. 2010. Antidepressant-Like Effect of Mitragynine Isolated from Mitragyna Speciosa Korth in Mice Model of Depression. Phytomedicine. IN PRESS,

[6] Z. Zhang. 2004. Therapeutic Effects of Herbal Extracts and Constituents in Animal Models of Psychiatric Disorders. Life Sci. 75: 1659-99.

[7] S. Kwon, B. Lee, M. Kim, H. Lee, H. J. Park, and D. H. Hahm. 2010. Antidepressant-like Effect of the Methanolic Extract from Bupleurum falcatum in the Tail Suspension Test. Psychiatry. 34: 265-270. 
[8] R. D. Porsolt, A. Bertin, and M. Jalfre. 1977. Behavioral Despair in Mice: A Primary Screening Test for Antidepressants. Archives Internationales de Pharmacodynamie et de Therapie. 229: 327336.

[9] L. Steru, R. Chemat, B. Thierry, and P. Simon. 1985. The Tail Suspension Test: A New Method for Screening Antidepressants in Mice. Psychopharmacology. 85: 367-370.

[10] D. G. Machado, L. E. B. Bettio, M. P. Cunha, J. C. Capra, J. B. Dalmarco, M. G. Pizzolai, and A. L. S. Rodrigues. 2009. Antidepressant-like Effect of the Extract of Rosmarinus Officinalis in Mice: Involvement of the Monoaminergic System. Progress in Neuro Psychopharmacology \& Biological Psychiatry. 33: 642-650.

[11] A. Modarresi Chahardehi, D. Ibrahim, and S. F. Sulaiman. 2009. Antioxidant activity and total phenolic content of some medicinal plants in Urticaceae family. Journal of Applied Biological Sciences. 2(3): 01-05.

[12] A. Modarresi Chahardehi, D. Ibrahim, and S. F. Sulaiman. 2009. Antioxidant, antimicrobial activity and toxicity test of Pilea microphylla. International Journal of Microbiology. doi:10.1155/2010/826830.

[13] A. S. Mellidis, V. P. Papageorgiou. 1993. Phenolic constituents from Onosma heterophylla. J. Nat. Prod. 56(6): 949-952.

[14] A. L. Eckeli, F. Dach, and A. L. S. Rodrigues. 2000. Acute Treatment with GMP Produces Antidepressant-Like Effects in Mice. Neuro Rep. 11: 1839-1843.

[15] A. D. E. Zomkowsi, A. O. Rosa, J. Lin, A. R. S. Santos, J. B. Calixto, and A. L. S. Rodrigues. 2004. Evidence for Serotonin Receptor Subtypes Involvement in Agmatine Antide- Pressant Like-Effect in the Mouse Forced Swimming Test. Brain Res. 1023: 256- 263.

[16] M. P. Kaster, I. Raupp, R. W. Binfaré, R. Andreatini, S. A. C. Rodrigues. 2007. Antidepressant-like Effect of Lamotrigine in the Mouse Forced Swimming Test: Evidence for the Involvement of Noradrenergic System. Euro. J. Pharmacol. 565: 119- 124.

[17] T. Deak, C. Bellamy, G. L. D`Agostino, M. Rosanoff, N. K. McElderry, and K. A. Bordner. 2005. Behavioral Responses During the Forced Swim Test are Not Affected by Anti-Inflammatory Agents or Acute Illness Induced by Lipopolysaccharide. Behavioural Brain Research.160: 125-134.

[18] J. F. Cryan, C. Mombereau, and A. Vassout. 2005. The Tail Suspension Test As A Model To Assessing Antidepressant Activity: Review of Pharmacological and Genetic Studies in Mice. Neurosci. Biobehav. Rev. 29: 571-625.

[19] B. Karolewicz, and I. A. Paul. 2001. Group Housing of Mice Increases Immobility and Antidepressant Sensitivity in the Forced Swim and Tail Suspension Tests. European Journal of Pharmacology. 415: 197-201.

[20] J. B. Harborne. 1998. Phytochemical Methods: A Guide to Modern Techniques of Plant Analysis. Third Edition. Chapman and Hall, UK. 292 - 293.

[21] J. Avalos, and H. I. Maibach. 2000. Dermatologic Botany. Library of Congress Cataloging in Publication Data. CRC Press LLC,

[22] V. K. Sharma, N. S. Chauhan, S. Lodhi, and A. K. Singhai. 2009. Anti-depressant Activity of Zizyphus xylopyrus. International Journal of Phytomedicine. 1: 12-17.

[23] A. Paulke, M. Schubert-Zsilavecz, and M. Wurglics. 2006. Determination of St. John's Wort Flavonoid-Metabolites in Rat Brain Through High Performance Liquid Chromatography Coupled with Fluorescence Detection. J. Chromatogr. B Analyt. Technol. Biomed. Life Sci. 832: 109-113.

[24] P. Willner. 1984. The Validity of Animal Models of Depression. Psychopharmacology. 83: 1-16.

[25] L. Bach-Rojecky, Z. Kalodera, and I. Samaržija. 2004. The Antidepressant Activity of Hypericum Perforatum L. Measured by Two Experimental Methods on Mice. Acta Pharm. 54: 157-162. 
[26] M. D. Shalam, S. M. Shantakumar, and M. L. 2007. Narasu. Pharmacological And Biochemical Evidence for the Antidepressant Effect of the Herbal Preparation Trans-01. Indian J. Pharmacol. 39: 231-234.

[27] J. F. Cryan, A. Markou, and I. Lucki. 2002. Assessing Antidepressant Activity in Rodents: Recent Developments and Future Needs. Trends Pharmacol Sci. 23: 238-245.

[28] B. Eisensamer, G. Rammes, G. Gimpl, M. Shapa, U. Ferrari, and G. Hapfelmeier. 2003. Antidepressants are Functional Antagonists at the Serotonin Type 3 (5-HT) Receptor. Mol. Psychiatry. 12: 994-1007.

[29] P. C. Shah, N. A. Trivedi, J. D. Bhatt and K. G. Hemavathi. 2006. Effect of Withania somniferona Forced Swimming Test Induced Immobility in Mice and its Interaction with Various Drugs. Indian J. Pharmacol. 50(4): 409-415.

[30] A. Carlsson, and M. Lindqvist. 1963. Effect of Chlorpromazine or Haloperidol on Formation of 3Mehoxytyramine and Normetanephrine in Mouse Brain. Acta Pharmacol et toxicol. 20: 140-144.

[31] W. F. Pirl, and A. J. Roth. 1999. Diagnosis and Treatment of Depression in Cancer Patients. In: Licino, J. and Wang, M. L., Biology of Depression, From Novel Insights to Therapeutic Strategies. 1: 378 . 